ÉGYPTE

monde arabe

\section{Égypte/Monde arabe}

25| 1996

Anthropologies de l'Égypte 2

\title{
Survivances antiques, comparatisme et diffusion
}

Remarques sur l'erreur cartographique dans la formation de l'aire culturelle méditerranéenne

Gilles Boëtsch et Jean-Noël Ferrié

\section{OpenEdition}

Journals

Édition électronique

URL : https://journals.openedition.org/ema/831

DOI : $10.4000 /$ ema.831

ISSN : 2090-7273

Éditeur

CEDEJ - Centre d'études et de documentation économiques juridiques et sociales

Édition imprimée

Date de publication : 30 mars 1996

Pagination : 51-68

ISSN : 1110-5097

Référence électronique

Gilles Boëtsch et Jean-Noël Ferrié, « Survivances antiques, comparatisme et diffusion », Égypte/Monde arabe [En ligne], 25 | 1996, mis en ligne le 08 juillet 2008, consulté le 07 juillet 2022. URL : http:// journals.openedition.org/ema/831; DOI : https://doi.org/10.4000/ema.831

Ce document a été généré automatiquement le 7 juillet 2022.

Tous droits réservés 


\title{
Survivances antiques, comparatisme et diffusion
}

\author{
Remarques sur l'erreur cartographique dans la formation de l'aire \\ culturelle méditerranéenne
}

Gilles Boëtsch et Jean-Noël Ferrié

1 Nous avons développé, dans de précédents travaux, la notion de comparatisme diachronique afin de désigner l'erreur qui consiste à comparer sommairement des faits actuels et des faits passés (Ferrié, 1993a ; Ferrié et Boëtsch, 1992a). Dans un premier temps, le comparatisme diachronique donne de la consistance à l'idée selon laquelle certains ensembles culturels et sociaux seraient stables dans le temps et dans l'espace. Il confond systématiquement analogie et identité (Ferrié, 1993b). Enfin, il désocialise les croyances et les pratiques, c'est-à-dire qu'il les traite comme des réalités substantielles, indépendamment des contextes sociaux dans lesquelles elles s'insèrent. Cette erreur est la conséquence directe de l'écrasement temporel introduit par l'idée même de comparer des choses semblables dans le temps : la comparaison n'est possible que si l'on considère que le temps n'en change ni le sens ni la pertinence. Nous avons ainsi affaire à une conception statique de la culture, qui est traitée davantage comme un objet que comme un fait de communication.

2 Cette réification a conduit l'anthropologie à de nombreuses errances : la classification raciale des groupes humains, la conception culturaliste de la culture et ce mélange des deux que représente la notion, faussement humaniste et faussement respectueuse des singularités, qu'est la notion d'ethnie. Malgré de judicieuses critiques (Amselle, 1990), ces erreurs continuent à rendre difficile une approche sereine de la culture, c'est-à-dire une approche conduite en termes de flux et de circulation plutôt qu'en termes d'appartenance à des entités stables. L'insistance actuelle à définir l'aire méditerranéenne comme une entité culturelle géographiquement localisée dans le bassin méditerranéen en est un exemple frappant.

3 Nous pensons, en effet, que la notion d'aire méditerranéenne ne va pas de soi, non plus que la notion floue de « Méditerranée » quand on prétend l'utiliser pour désigner une identité collective ou, tout au moins, une proximité particulière entre les habitants des 
deux rives, en fonction du fait qu'ils seraient les uns et les autres des Méditerranéens. En fait, ces notions paraissent davantage intégrées dans des dispositifs politiques entendus au sens large. Nous voulons dire par là - suivant une idée argumentée par Susan Ossman (à paraître) - que la géographie politique ancienne et contemporaine sert de fond de carte à l'établissement de l'aire culturelle méditerranéenne. L'origine de cette pratique peut être observée dans la littérature consacrée à l'ethnographie du Maghreb à l'époque coloniale. Suivant une interprétation que nous avions suggérée (Ferrie et Boëtsch, 1992a), nous allons tenter de montrer comment la construction de l'aire méditerranéenne a suivi une logique cartographique surdéterminant les faits culturels et sous-déterminant l'histoire contemporaine. Le comparatisme diachronique fut et demeure l'instrument de cette double erreur de détermination; les survivances en sont l'argument principal; quant au "diffusionisme», dont on a dit beaucoup de mal, il n'a pourtant rien à voir avec ces erreurs.

La succession des antiquités de référence

4 Si l'on ne tient compte que du début de la colonisation française du Maghreb, le modèle romain est apparu dès la conquête de l'Algérie comme un mode d'emploi (Thébert, 1978 ; Benabou, 1978,1980 ; Frémeaux, 1984) et de légitimation, lequel fut purement rhétorique au demeurant, puisqu'il servit seulement à présenter la colonisation sous un angle propre à conforter les topiques habituels du nationalisme français ${ }^{1}$. Il ne s'adressait pas aux indigènes ni ne sous-tendait les stratégies coloniales, il les enjolivait tout en permettant ainsi de penser l'Afrique du Nord à l'intérieur des cadres de référence habituels de la culture académique française. Les premières décennies de publication de la Revue africaine sont donc riches de comptes rendus de découvertes d'archéologie romaine.

5 Mais la France ne peut inventer qu'une romanité partielle puisqu'elle ne se présente pas elle-même comme tout uniment latine. Il lui faut prendre en considération son passé gaulois. C'est ce que suggère l'article de Féraud sur les «Monuments dits celtiques dans la province de Constantine » (Féraud, 1863). Selon lui, les mégalithes de cette région auraient été élevés par des légionnaires romains d'origine gauloise ${ }^{2}$. $\mathrm{Au}$ début du siècle, un anthropologue italien, Sergi, donna une interprétation romaine du peuplement européen (Sergi, 1901). Il décrivit l'aire d'extension de la «race méditerranéenne » depuis les rives sud de la Méditerranée jusqu'à la Grande-Bretagne (Ferrié et Boëtsch, 1992a, 1992b; Ferrié, 1994). Cette aire correspondait assez exactement à l'espace occupé par l'Empire romain. De fait, la théorie de la «race méditerranéenne » de Sergi s'opposait à la théorie aryenne ou germanique, alors en vogue, qui suggérait que le peuplement européen - porteur de la civilisation européenne - était d'origine nordique. La carte de l'Empire devenait ainsi la carte de l'aire méditerranéenne.

6 En fait, le modèle romain - quand bien même établissait-il déjà la centralité de la Méditerranée ${ }^{3}$ - contournait les civilisations indigènes du Maghreb, soit en maintenant une distinction colonisateurs/colonisés, soit en s'intéressant davantage aux prétendues migrations de peuples nordiques ou celtes qu'à leurs particularités culturelles. En effet, la notion d'aire culturelle méditerranéenne n'apparait qu'avec la prise en compte des civilisations antiques d'avant la période romaine, et tout d'abord avec le développement de l'archéologie égéenne (Evans, 1892-93,1897). Cette nouvelle profondeur historique permettait de penser l'unité méditerranéenne en tant que fait culturel et non plus comme conséquence d'une conquête. Par certains côtés, Sergi se 
rattache aussi à ce courant et ses arguments anthropologiques ${ }^{4}$ ont d'ailleurs servi à des spécialistes du monde égéen (Glotz, 1923). En montrant une Méditerranée sans domination, on montre aussi une Méditerranée partageant le même patrimoine, c'està-dire une Méditerranée qui peut être unie. Il est intéressant de constater que de cette orientation apparaît après la première guerre mondiale, se développe dans les années vingt (Maunier, 1922), trente (Benoît, 1930) et quarante (Benoît, 1943), aboutissant à l'étonnant livre de Jean Servier, Les Portes de l'Année, décrivant le monde berbère comme un monde antique perpétué, et publié l'année même où l'Algérie devient indépendante (Servier, 1962). Il est réellement difficile de ne pas considérer cette évolution comme une façon d'insister sur une appartenance commune, au moment même où l'évolution des nationalismes maghrébins établit une nette séparation entre les deux rives.

7 Ceci est, pour une part, un problème français. La véritable anthropologie sociale de l'aire méditerranéenne, totalement anglo-saxonne dans ses fondements (Davis, 1977 ; Pitt-Rivers, 1986), se développe, elle, essentiellement sur la Méditerranée du nord, dans les années cinquante, et ne s'intéresse que par raccroc aux sociétés arabes. Des études les concernant sont incluses dans les ouvrages collectifs dirigés par Peristiany et PittRivers (Peristiany, 1965,1968; Pitt-Rivers, 1963), mais ne modifient pas l'assiette de l'aire culturelle définie par les anthropologues. Même ceux d'entre eux qui font une large part au monde arabe dans leurs considérations globales sont, en fait et avant tout, des spécialistes de l'Europe (Gilmore, 1982,1987, qui travaille sur l'Espagne). Quant à l'ouvrage critique le plus récent et le plus novateur sur la question, il est conduit à partir de considérations sur un terrain grec (Herzfeld, 1987). Visiblement, les AngloSaxons ne construisent pas l'aire culturelle méditerranéenne à partir du même point de vue, et l'on est tenté de considérer que cela vient du fait qu'ils n'ont pas les mêmes conceptions géopolitiques que la France, Les «Méditerranées » de référence sont trop fluctuantes pour que l'aire soit prise en considération comme quelque chose qui va de soi.

Survivances, résistances et territoire

8 La principale façon de construire une continuité entre les rives de la Méditerranée a consisté à rechercher chez les Berbères les survivances de comportements préislamiques communs à d'autres sociétés méditerranéennes plus éloignées dans le temps. Nous passerons rapidement sur les arguments raciologiques retenus en faveur de cette hypothèse ; ils furent nombreux et nous en avons traité par ailleurs (Ferrié et Boëtsch, 1989,1991). Certains anthropologues pensaient que les Berbères venaient de la Baltique ; d'autres, reprenant l'hypothèse de Shaw (1734), déjà douteuse en son temps (Thomson, 1993), affirmaient qu'ils étaient d'origine berbère, d'autres d'origine gauloise ou écossaise. On comprend qu'un tel comparatisme eût d'évidentes limites, les mœurs observables se prêtant difficilement à d'aussi lointaines comparaisons.

Nous avons souligné l'importance du rôle tenu par le développement de l'archéologie égéenne dans le renversement du modèle romain. Mais il faut aussi tenir compte de trois autres causes.

10 La première est constituée par les développements de l'archéologie égyptienne ainsi que des travaux connexes sur les peuples libyques - parmi lesquels l'ouvrage de Bates, paru en 1912 (Bates, 1970). Les Libyans Notes de Randall-Maciver et Wilkin (1901) relatent une tentative, conduite à l'instigation de Flinders Petrie, pour rechercher des traces de l'influence égyptienne au Maghreb. Bien que les conclusions des auteurs 
démentent ce point de vue, il en demeure l'idée d'une continuité peuples/culture sur le pourtour méditerranéen, tout au moins en ce qui concerne le sud. Cette idée sera particulièrement développée par un savant local installé dans le beylicat de Tunis, Lucien Bertholon, le premier sans doute à la défendre de façon aussi systématique que brouillonne (Bertholon, 1904, 1913 ; Bertholon et Chantre, 1913).

11 La deuxième cause dont il faut tenir œmpte est le développement des études sur les croyances, les coutumes et les pratiques berbères et, plus précisément, celles qui avaient trait à la religion et qui, bien que revêtues la plupart du temps de formes islamiques, avaient peu à voir avec !a religion du Prophète. Les ethnographes de la période coloniale se sont toujours intéressés à celles-ci quoique, contrairement aux affirmations tendancieuses de Abdallah Hammoudi (1988), ils n'aient jamais tenté d'inventer une quasi-religion berbère ${ }^{5}$. Ils étaient seulement intéressés par les survivances antiques à l'intérieur de l'islam maghrébin, et - en dehors de ce qui avait trait à la magie comme à la sorcellerie - aux cultes agraires, aux cultes des saints ou au culte des grottes.

C'est cet intérêt qui allait permettre d'étendre le champ comparatif aux croyances et aux cultes attestés sur la rive septentrionale de la Méditerranée. Mais il faut se garder de l'isoler d'un mouvement plus large : l'intérêt pour le folklore et, plus largement, pour les « cultes naturistes ». On connait, bien sûr, l'influence des travaux de Frazer; encore faut-il en souligner l'impact sur les recherches de Doutté - principalement sur Magie et Religion dans l'Afrique du Nord, paru en 1908 (Doutté, 1984) - et, un peu plus tard, sur l'ethnographie du Maroc (Basset, 1925). Cependant, il convient surtout de souligner que cet intérêt local pour le folklore est contemporain au développement des études folkloriques, en Europe du Nord comme en Europe du Sud. On peut citer les nombreuses études de Saintyves (1936) entre les deux guerres ainsi que les travaux de Fernand Benoit, sur lesquels nous allons revenir. Cette concomitance n'est pas le fait du hasard. Elle permet surtout la constitution de corpus communs aux deux rives, et rend ainsi le comparatisme possible.

En s'intéressant aux survivances, il est assez facile d'envisager un monde méditerranéen anciennement uni et toujours dépositaire d'une civilisation commune. Mais ce mythe unitaire a des contreparties, à vrai dire assez désagréables. Ce sont des conséquences du comparatisme diachronique qui portent principalement sur la définition de l'identité culturelle. Nous savons maintenant - du moins quand nous parlons en tant qu'anthropologues - que les identités se construisent interactivement dans les situations de contact, c'est-à-dire qu'elles ne sont pas substantielles (Barth, 1969). Or, le comparatisme diachronique provient d'une conception substantiatiste de l'identité et, ce qui est pire, fait croire avec beaucoup de facilité qu'elle est naturelle. Prenons deux exemples se rapportant aux Touaregs. Dans The Eastern Libyans, Bates (1970) utilise vingt-neuf fois des faits ethnographiques rapportés par Duveyrier à propos des Touaregs, afin de décrire les moeurs des Libyens antiques. L'année qui suit, Gsell $^{6}$, dans son Histoire ancienne de l'Afrique du Nord, procède de la même façon, utilisant des descriptions contemporaines de Touaregs pour parler de l'Antiquité (Gsell, 1920,1929). Le danger de cette démarche est évident; elle suggère que les Touaregs d'aujourd'hui n'ont guère changé depuis les temps anciens, ce qui conduit à penser, en premier lieu, qu'ils se situent en quelque sorte hors du temps ou dans le passé et, en second lieu, que s'ils changent et cessent d'être « ce qu'ils ont toujours été », ils ne sont plus rien. 
14 En fait, le comparatisme diachronique considère que les traits culturels constituent l'identité transhistorique des peuples et sont, comme les crânes, des éléments stables de leur typification. Ce qui se perd, bien sûr, est la connexion avec l'histoire sociale des symboles. Mais allons plus loin. Le comparatisme diachronique tient pour établi que les peuples existent et sont seulement réductibles à eux-mêmes, c'est-à-dire qu'ils tiennent leur être de l'intangibilité d'un système symbolique et d'un groupe de pratiques afférentes, constitués à l'origine. En ce sens, on peut suivre la trace d'un peuple ou d'un groupe de peuples apparentés en étudiant la répartition des croyances. Selon cette conception, il y aurait des Méditerranéens parce que les mêmes croyances survivent de part et d'autre de la Méditerranée ${ }^{7}$. En d'autres termes, la notion de survivances permet de référer des croyances et des pratiques non à un intérêt actuel mais au patrimoine trans-historique d'une population, d'attester de la présence de caractéristiques culturelles tout en les excluant de la dynamique sociale, de faire ce qui a longtemps été le propre de l'anthropologie: affirmer que la culture «est le phénomène social fondamental et qu'elle culmine dans le sacré » (Bloom, 1987, p. 308). Les «survivances" sont une vieille idée de l'histoire des religions (Eliade, 1963, p. 197-232) et (malheureusement) aussi de l'anthropologie; elles sont inséparables des notions de «superstition» et de «magie» (Belmont, 1979, p. 54-55) comme de leur contenu péjoratif, dénotant une activité anormale, extraordinaire, excessive (ibid, p. 57).

$15 \mathrm{Du}$ point de vue cartographique évoqué au début de ce texte, les survivances permettent de procéder à des localisations. Contrairement à ce que pourrait suggérer une approche rapide de la place accordée, durant tout le XIX ${ }^{e}$ siècle, aux migrations - de l'étude desquelles l'anthropologie physique se fit longtemps une spécialité -, celles-ci racontent l'histoire de l'origine seulement pour préciser le statut des peuples une fois qu'ils sont stabilisés. Elles relatent une installation. Au contraire, les peuples en mouvement ne sont guère appréciés. Pour être repérables comme des entités discrètes et dotées de caractéristiques propres, ils doivent être territorialisés. Du fait que les cultures relèvent de l'identité des peuples, il en découle alors qu'elles doivent aussi être inscrites dans un territoire. On constatera ainsi que les survivances - c'est-à-dire le caractère rémanent des traits culturels d'une population - proposaient vis-à-vis de la culture une conception équivalente à la théorie de la résistance des types, en faveur chez les anthropologues physiques - c'est-à-dire la certitude du caractère rémanent des traits somatiques. La conjonction des survivances et de la résistance, si l'on peut ainsi dire, aboutit à nier que les groupes humains soient accessibles au changement. Les Berbères ont malheureusement bénéficié de cette négation. L'illustration la plus caricaturale en est donnée par Bertholon et Chantre qui, après avoir défini, dans les Recherches anthropologiques dans la Berbérie orientale (1913), les types somatiques de cette région comprenant l'Algérie, la Tunisie et la Tripolitaine, leur font correspondre des «tribus » décrites par les voyageurs de l'Antiquité8.

Mais qu'advient-il quand on souhaite établir une forme de proximité entre ces entités intangibles et territorialisées? Il est difficile d'adopter une attitude performative en ce qui les concerne, puisque les traits physiques, les traits corporels et leur territorialisation sont définis comme permanents. Les prémisses même de la question rendent impossible de la résoudre dans ce sens. Il y a, certes, l'hypothèse de l'acculturation et du métissage, mais cette hypothèse est trop étrangère aux conceptions rigides que nous venons d'évoquer pour être prise en considération. II 
reste à placer la proximité à l'origine, c'est-à-dire à envisager la carte des territoires peuples-cultures avant l'état actuel des choses, à situer le changement à l'origine, en d'autres termes, à tenir compte des contours de la carte et non de ses subdivisions territoriales. Ce qui ne signifie pas sortir de la géographie politique. On remarquera, par exemple, que la Méditerranée comme unité est toujours envisagée par rapport à l'extension des empires maritimes de la Crète, de la Grèce et de l'Asie Mineure ou de l'Empire romain.

17 Là aussi, nous avons affaire à une sélection : le fond de carte qui permet d'envisager l'unité est construit rétrospectivement, à partir de l'origine supposée de la civilisation européenne moderne. Indéniablement, la superposition de cette carte politique au bassin méditerranéen permet de la rendre naturelle, ainsi que l'a souligné David Kertzer" : "People subscribe to the "Master fiction" that the worid is divided into a fixed number of mutually exclusive nations; they see these units as part of the nature of things, and assume an antiquity that the nations in face back. » (Kertzer, 1988, p. 6) En même temps, la culture méditerranéenne se trouve dotée d'un espace naturel : celui de la Méditerranée. Or ces superpositions - espace naturel, espace politique, espace ethnique et espace culturel - relèvent tout simplement du mythe. C'est en cela que le comparatisme méditerranéen s'avère dangereux, car cette superposition est un faux-semblant.

Nous allons maintenant reprendre cette série de considérations en procédant à l'analyse d'un article publié en 1930 par Fernand Benoît dans la Revue anthropologique: "Survivances des civilisations méditerranéennes chez les Berbères ».

Les survivances méditerranéennes chez les Berbères, selon Benoît

19 Fernand Benoît est d'abord un spécialiste du folklore provençal, auquel il a consacré de nombreuses études. Il a aussi été maître de conférences à l'Institut des hautes études marocaines. Son premier article ${ }^{10}$ sur les survivances méditerranéennes débute ainsi : "L'intérêt du monde berbère est de nous transmettre vivant un monde disparu depuis trois millénaires. » (Benoît, 1930, p. 276). II poursuit ainsi : « La Méditerranée orientale, dont la puissance d'expansion fut si brillante à l'époque des civilisations mi-maritimes et mi-terrestres d'Asie Mineure et de Crète, nous donne en effet l'explication de monuments et de rites irréductibles à la civilisation romaine, qui ne fut pas la première à réaliser l'unité méditerranéenne. La Provence comme l'Ibérie du Sud, comme l'Afrique du Nord, fournissent autant d'arguments (...) en faveur d'une uniformisation des civilisations côtières de la Méditerranée. » (ibid., p. 279)

Ces considérations ne sont pas originales, ainsi que nous l'avons indiqué plus haut. Elles s'inscrivent, au contraire, dans un ordre d'idées constant, dont on peut esquisser le florilège en commençant par Bertholon: «L'an passé, J'ai essayé de vous montrer la voie courante des migrations venues sur le sol de la Tunisie moderne par le cabotage antique. Cette route qui passe par la Crète est jalonnée de noms qui rappellent les villes d'Asie mineure et aussi des cités grecques. Les légendes de la Grèce confirment ces relations. Le costume, la céramique, les pratiques agricoles, la religion des Libyens, la présence d'un stock considérable de termes européens dans les langues berbères, tout affirme l'importance d'une colonisation ancienne des peuples égéens dans le nord de l'Afrique. Un récent voyage en Grèce, la visite des collections préhelléniques des musées de ce pays, des survivances ethniques dans la population des campagnes grecques n'ont fait que fixer ma conviction. Je me suis demandé si l'organisation sociale des peuples de Berbérie différait beaucoup de celle des peuples antiques de la mer Egée. » (Bertholon, 1913, p. 190) 

lumière de l'esprit antique ne s'y éteint point C'est à la France de veiller à son maintien » (Maunier, 1930, p. 53), pour aboutir à Servier : "Les paysans de l'Algérie mieux que d'autres ont su longtemps garder ces valeurs intactes. Dans ce domaine, ils n'ont rien à attendre de l'Occident mais, bien au contraire, ils peuvent lui fournir les clés secrètes qui lui permettront d'ouvrir les héritages encore scellés de Rome, d'Athènes et de Mycènes. » (Servier, 1962, p. 14)

Mais pour Fernand Benoît, l'évocation de l'Antiquité s'inscrivait aussi dans un souci de méthode - le comparatisme diachronique dans le sens présent/passé ${ }^{11}$ - c'est-à-dire que la comparaison entre l'Antiquité et la période actuelle ne servait pas seulement, pour lui, à démontrer l'unité méditerranéenne (comme il le fera dans Benoît. 1943) mais également à promouvoir une méthode de recherche: "L'Afrique, où se sont conservés avec une étonnante vivacité des espèces et des rites disparus, nous fournit l'explication d'un monde périmé et peut nous être d'une grande utilité pour comprendre, par l'étude directe, des coutumes que nous ne connaissons que par les récits plus ou moins précis d'historiens de l'Antiquité. Sur le plan de la recherche générale, l'étude de l'ethnographique africaine rejoint l'étude des antiquités méditerranéennes. » (Benoît, 1930, p. 279) Dans cet article, Fernand Benoît ne traite pas de toutes les survivances mais s'intéresse aux rites saisonniers, et tout particulièrement à l'un d'entre eux : « Le mystère de la renaissance végétale, tel qu'il est célébré dans un grand nombre de fractions berbères de la montagne, nous est connu sous le nom bien mauvais de "nuit de l'erreur". (...) Cette nuit, qu'il serait plus scientifique d'appeler "nuit du renouveau", se différencie cependant des mystères antiques en ce sens que son symbolisme semble avoir perdu toute signification eschatologique.» (Benoît, 1930, p. 281)

La «nuit de l'erreur» consiste en une série d'accouplements collectifs entre des partenaires indistincts, lors du changement de saison, à l'automne la plupart du temps. Dans un premier temps, Benoît remarque l'analogie de cette pratique et des mystères d'Eleusis (ibid.); puis il repère des variantes euphémisées de la même pratique dans le monde berbère. Il s'agit d'abord des processions symboliques des poupées de la mariée : dans le Sous, « les jeunes filles sortent processionnellement portant un bâton avec bras transversal que drapent des vêtements féminins » (ibid., p. 282). Viennent ensuite, cités d'après Laoust (1920), des cas où jeunes gens et jeunes filles sont unis «plus ou moins symboliquement » dans la mosquée du village (ibid.). Des unions réelles succéderaient à ces unions symboliques. Il relève des descriptions semblables chez des auteurs antiques, notamment chez Nicolas de Damas qui aurait signalé la "nuit de l'erreur » chez les Dapsolibyens du sud de la Tunisie (ibid., p. 283). À ce propos, il reproche à Bertholon (1898) d'avoir rapproché ces faits des mœurs attribuées par Strabon aux Amazones du Caucase (ibid., note 4). Il cite ensuite Léon l'Africain puis Mouliéras, évoque les orgies célébrées chez les Béni Mahsen et revient aux mystères d'Eleusis : «Le mystère d'Eleusis se terminait au matin par un rite qui achève d'expliquer la nuit grecque; le hiérophante, rallumant tes torches éteintes, présentait symboliquement l'épi de blé en disant ces paroles: "La divine Brimo a enfanté Brimos". Ainsi la nuit figurait le cycle du mystère sacré, dont les phases étaient l'union rituelle, la fécondité et la génération. » (Benoît, 1930, p.286).

Ayant ainsi établi l'identité de la «nuit de l'erreur» chez les Berbères et chez les anciens Grecs, Benoit étend la comparaison à d'autres points du bassin méditerranéen

Égypte/Monde arabe, 25 | 1996 
et, notamment, à l'Espagne et à la Provence : «Un développement parallèle des rites, s'il était possible de le prouver, apporterait un argument de plus au postulat que j'ai posé au début, à savoir que l'Afrique berbère nous a transmis l'état d'un milieu datant de trois millénaires.» (Ibid., p. 287) II relève des cérémonies comparables à la « nuit de l'erreur " dans certaines parties montagneuses de la Provence et du Dauphiné, dont nous avons eu connaissance par les Inquisiteurs du Saint-Office. Ces pratiques relevaient, selon lui, du manichéisme cathare "qui avait refleuri en Gaule (...), et principalement dans les Alpes du $\mathrm{XI}^{\mathrm{e}}$ au $\mathrm{Xv}^{\mathrm{e}}$ siècle, et du Vaudisme" (Ibid., p. 288). Benoît note ensuite le parallélisme unissant les mystères d'Eleusis, la «nuit de l'erreur " chez les Béni Mahsen et les assemblées nocturne chez les Vaudois; «Le Vaudois qui présidait à ce mystère, dans la maison de quelque habitant du village où ils se réunissaient, éteignait la lumière et prononçait la phrase rituelle : qui habet habeat ou teneat, traduction littérale de la phrase entendue dans la caverne des Béni Mahsen et signal de l'orgie. Comme il faut bien penser que l'enquêteur marocain ne connaissait pas les procès d'inquisition des Vaudois et que ces inquisiteurs n'étaient pas au courant des mystères d'Eleusis, on ne peut qu'être frappé de ce parallélisme, sans oser en conclure que les "nuits vaudoises" se rattachent à des mystères agraires de l'Antiquité... » \{Ibid., p. 288-289)

25 Fernand Benoît cite, pour conclure, des pratiques qu'il juge similaires en Provence, bien qu'elles n'impliquent pas d'orgie: «Les villageois, non contents de porter en procession la statue d'un saint dont la vertu agricole fait toute la force, passent ensemble une nuit dans un oratoire à l'occasion de cette procession. » (ibid, p. 289)

Limites et diffusions dans la définition de l'aire méditerranéenne

Nous venons de voir comment Fernand Benoît procédait; il établissait d'abord une analogie entre des pratiques actuelles observables chez les Berbères et une pratique attestée dans l'Antiquité, puis en notait l'extension et les variantes sur le pourtour méditerranéen. Il soutenait l'hypothèse selon laquelle les assemblées nocturnes liées à des cultes agraires seraient un invariant méditerranéen. Toutefois, on peut observer qu'il procédait avec une grande prudence et beaucoup de réserves. Il relevait des analogies, soulignait la ressemblance de faits contemporains et de faits antiques mais ne soutint jamais positivement une hypothèse sur leur filiation :

«On ne peut qu'être frappé par ce parallélisme, sans oser conclure...», écrit-il d'ailleurs. Si l'on compare cette façon de faire à celle de Bertholon qui soutenait, lui, des thèses diffusionnistes, on est d'abord porté à la satisfaction. Il existe une aire méditerranéenne parce que les mêmes traits culturels sont partagés sur le pourtour de la Méditerranée, et il ne sert à rien de démontrer la filiation ou l'identité pour établir ce fait. La façon de voir de Fernand Benoît est contemporaine d'une rupture épistémologique qui a consisté, dans l'étude des sociétés riveraines de la Méditerranée, à ne plus forcément confondre analogie et identité, comme on l'avait fait jusqu'alors sous l'influence de l'anthropologie physique, qui considérait que les analogies dans les traits culturels provenaient de l'origine commune des populations chez lesquelles on les trouvait (Ferrié, 1994b).

Il est, certes, louable de sortir de la stricte liaison peuple/culture, mais la position prudente et floue de Benoît n'est guère satisfaisante non plus, car il reste à expliquer les analogies. Or ceci ne va pas de soi. En fait, si l'on excepte les biais et les erreurs dans l'évaluation des faits ethnographiques, il est difficile d'affirmer la dissémination d'une même pratique sans envisager un processus de diffusion, c'est-à-dire une origine 
commune. Comme il ne possède pas de fil conducteur, Benoît évite de conclure en ce sens. Pourtant, cette hypothèse est en quelque sorte affirmée quand il évoque le «moule du monde classique (...), cette civilisation préhellénique et préromaine des peuples égéo-crétois" (Benoît, 1930, p. 279) et les mystères d'Eleusis. Ici, le comparatisme diachronique introduit à l'évidence une idée de l'origine.

On remarquera alors que l'origine du local est toujours locale, même s'il s'agit d'un local étendu dans l'espace et dans le temps. On élargit le cadrage cartographique mais l'on évite de recadrer. Or, est-il sûr que les «assemblées nocturnes » se prêtent à la démonstration de l'unité méditerranéenne? Benoît a cité des faits rapportés par les inquisiteurs, qui se déroulèrent dans les Alpes et le Dauphiné; ces faits - des assemblées nocturnes suivies d'orgies - sont cependant attestés en Europe hors de la zone méditerranéenne. Carlo Ginzburg a montré que leur géographie impliquait un cadrage assez différent, puisqu'on les retrouvait en Europe du Nord et de l'Est et jusqu'en Sibérie (Ginzburg, 1992). On peut, certes, être en désaccord avec telle ou telle partie de la thèse de Ginzburg; en revanche, elle indique qu'il existe une autre façon d'envisager la distribution géographique des assemblées nocturnes et que cette distribution n'est pas centrée sur le bassin méditerranéen. Or, si l'on suit la bibliographie de Benoit (Dollinger, 1890), il est permis de douter qu'il ait pu ignorer la répartition européenne des "assemblées nocturnes ». Mais celles-ci, comme beaucoup d'autres faits prétendument "méditerranéens » - le mauvais œil ou la mise en jeu de l'honneur de l'homme par la honte de la femme, par exemple - ne le sont, en définitive, que parce qu'on coupe le reste de la carte de leur localisation. En d'autres termes, parce que le cadrage méditerranéen l'emporte sur les autres cadrages possibles. En ce sens, les faits sont surdéterminés, et sans doute la construction sociale de l'origine grécolatine de l'Europe (et de la France) y est-elle pour beaucoup, car cette origine sous-tend visiblement toutes les comparaisons méditerranéennes, tout au moins jusqu'à ce que l'anthropologie sociale anglo-saxonne s'y intéresse.

30 Nous venons d'indiquer d'autres cadrages abusifs comme le mauvais œil - qui existe aussi en Afrique de l'Est et en Inde - ou la mise en jeu de l'honneur de l'homme par la honte de la femme. On pourrait aussi parier de la vengeance et des histoires de familles: les drames de Shakespeare (ceux qui n'ont pas l'Italie pour décor) nous invitent à penser que la Méditerranée n'a pas le privilège de ces fureurs - du clientélisme, non plus; et sans doute serait-il possible de comparer le clientélisme chinois ou vietnamien au clientélisme italien, maltais ou marocain. Inversement, si l'on prend en compte ces mêmes traits sans s'embarrasser de les faire cadrer avec le bassin méditerranéen, peut-être faudrait-il alors étendre l'aire culturelle méditerranéenne à l'Amérique latine et à une part non négligeable des Etats-Unis. En fait, nous ne sommes pas sûrs de nos cartes culturelles ni de l'identité des traits distinctifs que nous utilisons pour les tracer, et rien n'indique enfin que les superpositions auxquelles nous nous livrons soient autre chose que des cadrages abusifs influencés par la façon dont nous construisons mythiquement notre propre identité.

31 Le comparatisme méditerranéen, comme les comparaisons avec l'Antiquité, sont aussi séduisantes que trompeuses. Il semble pourtant que l'idée de Méditerranée soit aujourd'hui très en faveur dans les sciences sociales françaises, sans que son contenu paraisse pour autant bien clair. La notion de Méditerranée permet-elle, par exemple, de comprendre ce qu'il y a actuellement de commun entre Milan, Rome, Tunis, Athènes, Tanger ou Le Caire? Et ce que l'on y retrouve de semblable vient-il d'une ancienne 
culture partagée ou, plus simplement, du fait que la même culture transnationale - qui n'est pas exactement méditerranéenne - y est à l'œuvre ? De plus, ne confond-on pas identité substantielle et identité performative? Une unité culturelle existe-elle objectivement en dehors de sa prise en considération effective par les membres des diverses sociétés qui pourraient s'en réclamer? Les habitants de la rive sud ne se décrivent pas comme des "Méditerranéens" mais comme des "Arabes». Ce sont principalement les Européens qui parlent de Méditerranée (ou les Marocains quand il s'agit des relations avec l'Europe), et ce fait n'est pas négligeable. Le dernier mauvais service que peut nous rendre la notion de Méditerranée est de perpétuer cette erreur fréquente du sens commun et, trop souvent encore, du sens anthropologique selon laquelle l'origine d'une croyance ou d'une pratique serait dotée de signification. Or les croyances et les pratiques, les symboles, n'ont de sens que par leur usage. La chose a été dite et redite puis posée avec beaucoup de clarté par Wittgenstein (1953, p. 128). Si les Marocains ou les Égyptiens ne se définissent pas comme Méditerranéens, ils ne le sont pas, et leur trouver des traits méditerranéens n'y changera rien. De plus, cela oriente certainement dans un mauvais sens la recherche des proximités entre "eux " et "nous ». Nous les situons dans des choses passées et non dans des choses présentes: dans le partage, par exemple, de la même conception de l'honneur et non dans l'écoute commune de "Dallas $»^{12}$. Ce qui suggère à tort que les particularités et les proximités se jugent sur le partage des « archaïsmes » et non sur l'utilisation différenciée de la même modernité13.

Certes, si l'on se promène au Caire, beaucoup de façades du centre-ville nous évoquent l'Italie, mais les Cairotes font-ils la même comparaison? Et Casablanca est-elle une ville méditerranéenne ou atlantique? L'impossibilité de répondre de façon satisfaisante à des questions aussi anodines suggère que notre idée de la Méditerranée n'est guère plus pertinente que ne l'était notre idée de l'Orient.

\section{BIBLIOGRAPHIE}

Abu-Lughod L, « Bédouins, Cassettes and Technologies of Public Culture », Middie EastReportn' 159, 1989, p. 7-11.

Amselle J.-L., Logiques métisses (Anthropologie de l'identité enAfrique et ailleurs), Payot, Paris, 1990.

Anderson B., Imagined Communities: Reflections on the Origin and Spread of Nationalism, Verso, Londres, 1983.

Barth F., Ethnic Groups and Boundaries, Uttle, Brown and Co., Boston, 1969.

Basset R., « Recherches sur la religion des Berbères », Revue de l'Histoire des Religions, 1910, p. 291-342.

Basset H., « Compte rendu de Frazer, Le Rameau d'or », Hesperis, t. V, 1925, p. 377-379.

Bates O., The Eastern Libyans, An Essay, Franck Cass \& Co., Londres, 1970 (1912). 
Belmont N., « Superstition et religion populaire dans les sociétés occidentales », La Fonction symbolique (Essais d'anthropologie), M. Izard et P. Smith éd., Gallimard, Paris, 1979, p. 53-70.

Benabou M., "L'impérialisme et l'Afrique du Nord : le modèle romain », Sciences de l'Homme et conquête coloniale. Constitution et usage des sciences humaines en Afrique (XIX ${ }^{e}-\mathrm{XX}$ ), 0. Nordman \& J.-P. Raison éd., Presses de l'École normale supérieure, Paris, 1980,

Benoît F-, «Survivances des civilisations méditerranéennes chez les Berbères », Revue anthropologique t. XL, nº 7-9,1930, p. 278-293.

- « L'Unité méditerranéenne », Cahiers du Sud (numéro spécial : Le Génie d'Oc et l'Homme méditerranéen), 1943.

Bertholon L., " Origine néolithique et mycénienne des tatouages des indigènes du nord de l'Afrique ", Archives d'anthropologie criminelle, de criminologie et de psychologie normale et pathologique $\mathrm{n}^{\circ} 130,1904$, p. 1-31.

- «Sociologie comparée des Achéens d'Homère et des Kabyles contemporains ", Revue tunisienne, 1913.

Bertholon L. et Chantre E., Recherches anthropologiques dans la Berbérie orientale, Tripolitaine, Tunisie, Algérie, Rey, Lyon, 1913.

Bloom A., L'Âme désarmée. Essai sur le déclin de la culture, Julliard, Paris, 1987.

Boëtsch G. et Ferrié J.-N., « Le Paradigme berbère. Approche de la logique classificatoire des anthropologues français du XIX siècle ", Bulletins et Mémoires de la Société d'anthropologie de Paris, vol. 1 (nelle série), n 3-4, 1989, p. 257-276.

- « Blonds (berbères) », Encyclopédie berbère, t. X, Edisud, Aix-en-Provence, 1991.

Boissière G., L'Algérie romaine, Hachette (2e édition), Paris, 1883 (1875).

Coye N., «Préhistoire et protohistoire en Algérie au XIXe siècle, les significations du document archéologique ", Le Savant et le Berbère, Cahiers d'études africaines $\mathrm{n}^{0} 129$, G. Boetsch \& J.-N. Ferrie éd., 1993, p. 99-137.

Davis J., The People of the Mediterannean: An Essay in Comparative Social Anthropology, Routledge \& Kegan Paul, Londres, 1977.

Dollinger W., Be/frage zur sektengeschichte des Mifielalters. Munich, 1890.

Doutté E., Magie et religion dans l'Afrique du Nord (La société musulmane du Maghreb), Maisonneuve et Geuthner, Paris, 1984 (1908).

- Les Tas de pierres sacrés et quelques pratiques connexes dans le sud du Maroc, Alger, Heintz, s.d.

Eliade M., Aspects du Mythe, Gallimard (collection Idées), Paris, 1963.

Evans A., "Primitive Pictographs and pre-Phœnician Script from Crete », The Journal of Hellenic Studies, XIV, 1894, p. 270-296.

Evans A., « Further Discoveries of Cretan and Egean Script: with Ubyan and Proto-Egyptian Comparisons ", The Journal of Hellenic Studies, XVII, 1897, p. 327-395.

Féraud C., " Monuments dits celtiques dans la province de Constantine ", Bulletin de la Société archéologique de la Province de Constantine n ${ }^{\circ} 7,1863$, p. 214-234,

Ferrié J.-N., « Le comparatisme diachronique et l'ethnographie touarègue », Ethno-histoire du monde touareg. Réseaux et formations politiques, Claudot-Hawad éd., Aix-en-Provence, Iremam, 1993a. 
- « La naissance de l'aire culturelle méditerranéenne dans l'anthropologie physique de l'Afrique du Nord », Le Savant et le Berbère. Cahiers d'Etudes africaines n 129, G. Boëtsch \& J.-N. Ferrie éd., 1993b, p. 139-151.

- «Sciences coloniales, centralité scientifique et périphérie savante. Le Maghreb et l'Egypte comme applications locales d'un discours global », Annales islamologiques t. 27, 1994.

- Ferrié J.-N, et Boëtsch G., « Du Berbère aux yeux clairs à la race eurafricaine, la Méditerranée des anthropologues physiques ", Le Maghreb, l'Europe et la France, K. Basfao \& J.-R. Henry éd., Presses du CNRS, Paris, 1992a.

- Ferrié J.-N. et Boëtsch G., « La Formation de l'aire culturelle méditerranéenne par les anthropologues européens du XIX siècle ", Antropologia Contanporanea, vol. 15, nº 1,1992b, p. 75.81 .

Frémeaux J., " Souvenir de Rome et présence française au Maghreb : essai d'investigation », Connaissances du Maghreb, Sciences sociales et colonisation, J.-C. Vatin éd., CNRS, Paris, 1984.

Gilmore D., " Anthropology of the Mediterranean area ", Annual Review of Anthropology r ${ }^{\circ} 11,1982$, p. 175-205.

-Aggression and Community: Paradoxes of Andalusian Culture, Yale University Press, New Haven, 1987.

Ginzburg C., le Sabbat des sorcières (trad. de Il Sabbato, Einaudi, Turin, 1989), Gallimard, Paris, 1992.

Glotz, La civilisation égéenne, La Renaissance du livre, Paris. 1923.

Gsell S., Histoire ancienne de l'Afrique du Nord. 1.1, Paris, 1920 (1913).

- Histoire ancienne de l'Afrique du Nord. t. IV. Paris, 1929 (1913).

Hammoudi A., La victime et ses masques, Seuil, Paris, 1988.

Herzfeld M., Anthropology through the Looking-Glass. Critical Ethnography in the Margins of Europe, Cambridge University Press, Cambridge, 1987.

Kertzer D., Ritual. Politics and Power, Yale University Press, New Haven, 1988.

Laoust E., Mots et choses berbères, Challamel, Alger, 1920.

Maciver R. et Wilkin A, Libyan Notes, Macmillan, Londres, 1901.

Maunier R., Cours de sociologie algérienne, Hespéris, t. 2 ( $1^{\text {er }}$ et $2^{\mathrm{e}}$ trim.), 1922.

Maunier R., Mélanges de sociologie nord-africaine, Alcan, Paris, 1930.

Ossman S., « Bob Marley entre nous deux? », Les Relations interculturelles, Y. Winkin éd., à paraître.

Peristiany J. (éd.), Honor and Shame: the Values of Meiterranean Society, Weidenfeld \& Nicolson, Londres, 1965.

- Contributions to Mediterranean Sociology, Mouton, La Haye, 1968.

Pitt-Rivers J., Mediterranean Countrymen: Essays in the Sociology of the Mediterranean, Paris. Mouton, 1965.

Rogers S., Shaping Modem Times in Rural France, Princeton University Press, Princeton, 1991.

Roussillon A., « Le partage des savoirs. Antériorité de l'image coloniale en Egypte », Annales islamologiques, t. 26,1992, p. 207-249.

Saintyves P., Manuel de folklore, Nourry, Paris, 1936. 
Sergi G., The Mediterranean Race: A Study of the Origin of European Peoples, Waller Scott, Londres, 1901.

Servier J., Les Portes de /'Année. L'Algérie dans la tradition méditerranéenne, Laffont, Paris, 1962.

Shaw T., Travels or Observations Relating to Several Parts of Barbary and the Levant, Oxford, 1734.

Thébert $\mathrm{Y}$, « Romanisation et déromanisation en Afrique, histoire décolonisée ou histoire inversée », Annales ESC, vol. 33, n 1,1978, p. 64-82.

Thomson A., "La classification raciale de l'Afrique du Nord au début du XIX siècle ", Le Savant et le Berbère. Cahiers d'Etudes africaines n 129, G. Boëtsch \& J.-N. Ferrié éd., 1993, p. 19-36.

WainwrightG., « Ethnology in Egypt », Bulletin de la Société khédiviale de géographie du Caire, vol. XVI, $n^{\circ} 4,1929$.

Westermarck E., Survivances païennes dans la civilisation mahométane (trad. de Pagan Survivals in Mohammedan Civilisation, Londres, 1933), Payot, Paris, 1935.

Wittgenstein L., Philosophical Investigations, Blackwell, Oxford, 1953.

\section{NOTES}

1. Prenons, par exemple, Gustave Boissière, latiniste, recteur de l'Académie d'Alger dans le dernier quart du XIX siècle. Il écrit dans l'avant-propos, daté de 1875, de L'Algérie romaine: « Dépouillé aujourd'hui de notre vieil empire colonial, mais amenés par un dernier bonheur ou par une dernière habileté sur ce littoral africain, jadis si peuplé, si prospère, paré des monuments et des efforts de trois civilisations successives, puis longtemps désolé par la barbarie, nous nous sommes imposé la tâche d'en régénérer, d'en relever la race, d'y rapporter le travail, la richesse et la vie. (...) Aussi bien, s'il est vrai qu'il fût exagéré de dire des indigènes de l'Afrique française ce que Tacite disait des provinces, qu'elles se soumettaient de bon cœur au nouvel ordre des choses, il semble cependant que, frappés sinon réconciliés par la ressemblance de notre œuvre civilisatrice avec l'œuvre romaine. dont l'histoire leur parle vaguement, dont surtout ils retrouvent à chaque pas les traces, ces hommes qui nous appellent des roumis se résignent à subir en nous les légataires de Rome. » (Boissière, 1883, p. xv-xvi)

2. Sur ces monuments et les différentes théories qui s'y rapportaient, voir Coye (1993), Boëtsch et Ferrié (1991) et Ferrié et Boëtsch (1992a).

3. À ce propos, Boissière écrivait : « [la Méditerranée] jadis infestée qui baigne ces rivages [de l'Algérie] et les rivages de France et qui, les séparant naguère, ne fait plus que les rapprocher et les réunir aujourd'hui » (Boissière, 1883, p. xv-xvi) ; et : « ...dans une colonie si voisine qu'elle est proprement (...) le prolongement de la métropole au-delà de ce lac jadis romain et maintenant français qui sépare Alger et Marseille... » (Ibid., p, xxix)

4. Nous entendons par là l'anthropologie physique.

5. Et il n'y eut jamais de présentation systématique d'une telle religion, y compris chez Basset (1910). Des articles furent, certes, publiés sur des croyances, des cultes ou des rites spécifiques et leur origine antique. Certains s'avèrent particulièrement intéressants comme Les tas de pierres sacrés (Doutté, s.d.) ou Le Culte des grottes au Maroc (Basset, 1920), mais ne prétendent pas aller audelà de la permanence de certaines croyances spécifiques. Pour Doutté, il ne s'agit même pas, dans Les Tas de pierres sacrés, de prouver la survivance d'anciennes formes religieuses mais d'étudier leur islamisation : « Notre but principal n'était pas, comme on pourrait le croire, de montrer que l'Islam s'est assimilé des pratiques païennes antérieures à lui ; on sait bien qu'il en 
est ainsi de toutes les religions et la continuité des cultes est devenue une thèse banale. (...\} Ce qui est intéressant, c'est de rechercher par quel procédé chaque religion s'est appropriée et s'est assimilée le fond commun des antiques conceptions sauvages et comment elles ont évolué dans la suite. Nous avons voulu surtout faire ressortir par un exposé systématique comment l'Islam s'est servi du maraboutisme pour s'assimiler les rites magiques chers à l'imagination des populations magribines (sic).» (s. d., p. 37-38).

6. Gsell (1920, p. 244-245; 1929, p. 125). Ces exemples sont présenté et mis en contexte dans Ferrié (1993a).

7. Ce point de vue est défendu par Westermack (1935\}; voir aussi Ferrié ( 1993b).

8. Cette question est traitée dans Boëtsch et Ferrié (1989).

9. Voir aussi (et surtout) Anderson (1983).

10. Le second fut publié treize ans plus tard dans les Cahiers du Sud (1943).

11. On remarquera un souci semblable, mais dans le sens passé/présent, en ce qui concerne l'Égypte : «S'il y a quelque intérêt à s'interroger sur le service spécifique que l'Égypte est la plus désignée pour rendre à cette forme de savoir..., demandons-nous quel pays est mieux à même de manifester, à travers des siècles de civilisation, la permanence d'habitudes, d'arts et de métiers à travers leur évolution. De ce point de vue, l'ethnologie permettra la comparaison entre les coutumes des anciens Égyptiens et celles du temps présent, telles qu'elles se perpétuent jour après jour, heure après heure autour de nous sans que nous nous en avisions. » (Wainwright, 1929 , p. 258, traduit et cité par Roussillon, 1992, p. 223).

12. Sur ce point, voir Abu-Lughod (1989).

13. Voir le développement subtil de ce point de vue dans Rogers, 1991, p. 207-214.

INDEX

Mots-clés : cartographie, anthropologie, Méditerranée, comparatisme

\section{AUTEURS}

GILLES BOËTSCH

CNRS

JEAN-NOËL FERRIÉ

Cedej 